Patrick Gosteli MD, Zdravko Gamulin MD, Elizabeth Van Gessel MD, Alain Forster MD

\title{
Anesthésie péridurale pour la chirurgie de la cheville et du pied : influence de la position assise
}

\begin{abstract}
Dans ce travail, les effets de la position assise sur la qualité du bloc sensitivo-moteur des racines $L_{5}$ et $S_{1}$ lors d'une anesthésie péridurale et ses répercussions hémodynamiques ont été étudiés chez 39 patients subissant une chirurgie orthopédique de la cheville ou du pied. Après la mise en place d'un cathéter péridural en position latérale, 19 patients furent placés en position assise pendant 15 min après administration de l'anesthésique local et 20 en décubitus dorsal (groupe contrôle). Tous ont reçu une dose de $20 \mathrm{ml}$ de lidocaïne $1,73 \%$ carbonatée adrénalinée. Le temps d'installation et la qualité du bloc sensitif des racines $L_{I}-S_{2}$ ainsi que les niveaux céphaliques sensitifs ont été comparables dans les deux groupes. Dans le groupe assis, 14 patients sur 19 ont présenté un degré de bloc moteur $\geq 3 / 5$ contre 5 sur 20 dans le groupe couché $(P<0,001)$. La diminution de la tension artérielle moyenne a été plus importante dans le groupe assis $(-24 \pm 10 \%)$ comparativement au groupe couché $(-16 \pm 10 \% ; P<0,05)$ et a été observée plus précocément $(14 \pm 9$ min et $21 \pm 10$ min respectivement; $P<0,01)$. En conclusion, les résultats de cette étude indiquent que la position assise pendant 15 min lors d'une anesthésie péridurale ne modifie en rien la qualité du bloc sensitif, mais permet d'améliorer significativement la qualité du bloc moteur.
\end{abstract}

The effects of the sitting position on the quality of both sensory and motor blockade of segments $L_{5}$ and $S_{1}$ and the haemodyna-

Key words

ANAESTHESIA: orthopaedic;

ANAESTHETIC TECHNIQUES: peridural;

POSITION: effects;

ANAESTHETICS, LOCAL: lidocaine.

Département d'Anesthésiologie, Hôpital Cantonal

Universitaire, 1211 Genève 4, Suisse.

Adresse pour la correspondance: Dr P. Gosteli, Département d'Anesthésiologie, Hôpital Cantonal Universitaire, 1211 Genève 4, Suisse.

Accepted for publication 14th December, 1991. mic consequences during epidural anaesthesia were studied on 39 patients undergoing ankle or foot surgery. After insertion of an epidural catheter with the patient in the lateral position, 19 patients were kept sitting for 15 min following the injection of the local anaesthetic and 20 remained supine for the duration of anaesthesia (control group). All patients received a dose of $20 \mathrm{ml}$ of $1.73 \%$ carbonated lidocaine with epinephrine 1:200,000. The quality and time of onset of the sensory blockade for segments $L_{1}-S_{2}$ as well as its cephalad spread were comparable in both groups. Fourteen patients of the sitting group achieved motor blockade of more than three of five myotomes compared with five patients in the supine group $(P<0.001)$. The maximum decrease in mean arterial pressure occurred sooner in the sitting group $(14 \pm 9 \mathrm{~min})$ than in the control group $(21 \pm 10 \mathrm{~min} ; P<0.01)$ and was more severe $(-24 \pm 10 \%$ vs $-16 \pm 10 \%$ respectively; $P<0.05)$. Our results indicate that placing the patient in the sitting position for $15 \mathrm{~min}$ after inducing epidural anaesthesia does not influence caudal sensory blockade but does increase the depth of motor blockade.

Lors de l'anesthésie péridurale, la difficulté d'obtenir un bloc sensitivo-moteur de bonne qualité des racines de grand diamètre, c'est-à-dire $L_{5}$ et $S_{1}$, limite l'utilisation de cette technique pour les interventions de la cheville et du pied. ${ }^{1,2}$ Galindo $^{3}$ a rapporté un taux d'échec du bloc sensitif de $46 \%$ pour la racine $S_{1}$ avec l'hydrochlorure de lidocaïne adrénalinée à 1:200 000 chez des patients âgés de 14-40 ans. L'introduction de la lidocaïne carbonatée en anesthésie péridurale, comparativement à l'hydrochlorure de lidocaïne, a permis d'améliorer, d'après certains auteurs, la vitesse d'installation et la qualité du bloc sensitivo-moteur, pour les racines $L_{5}$ et $S_{1}$ en particulier. ${ }^{4-6}$ Toutefois d'autres études ont montré que seul le bloc sensitif ${ }^{7}$ ou seul le bloc moteur ${ }^{8}$ est amélioré. Quant à Cole, ${ }^{9}$ il n'a démontré aucune différence entre ces deux préparations. La position assise lors de l'injection de l'anesthésique local pourrait influencer favorablement la qualité du bloc des racines $\mathrm{L}_{5}$ et $\mathrm{S}_{1} \cdot{ }^{2}$ Cependant, les résultats de plusieurs études sur les effets de cette position 
lors de l'anesthésie péridurale sont contradictoires. ${ }^{1,2,4,10-15}$ A notre connaissance, aucune étude n'a comparé l'influence de la position assise sur la qualité du bloc moteur lorsqu'une chirurgie de la cheville ou du pied était réalisée. Le but de cette étude était donc d'étudier l'influence de la position assise sur la qualité du bloc sensitivo-moteur des racines $L_{5}$ et $S_{1}$ lors d'une anesthésie péridurale avec la lidocaïne carbonatée, pour la chirurgie de la cheville et du pied.

\section{Méthode}

Après acceptation par le Comité d'éthique de notre institution, 40 patients âgés de 20 à 50 ans, ASA 1 ou 2, programmés pour une intervention programmée de la cheville ou du pied sous anesthésie péridurale ont donné leur consentement oral. Les femmes enceintes et les patients ayant une taille inférieure à $1,5 \mathrm{~m}$ ou supérieure à $2 \mathrm{~m}$ ont été exclus. Les patients ont été divisés en deux groupes de 20 de façon randomisée : dans le groupe 1, la position couchée fut conservée pendant toute la durée de l'anesthésie ; dans le groupe 2 , la position assise à 90 degrés fut maintenue pendant 15 min à compter de l'injection de l'anesthésique local. Une heure avant l'intervention, tous les patients ont reçu une prémédication composée de péthidine $1 \mathrm{mg} \cdot \mathrm{kg}^{-1}$ et de prométhazine $0,3-$ $0,5 \mathrm{mg} \cdot \mathrm{kg}^{-1}$, en injection intramusculaire. La fréquence cardiaque a été mesurée en continu à l'aide d'un monitoring ECG et la tension artérielle déterminée toutes les 2,5 min par un appareil automatique non invasif (Minimap) pendant $45 \mathrm{~min}$, puis toutes les $5 \mathrm{~min}$, jusqu'à la fin de l'intervention. Après avoir mis en place une voie veineuse périphérique, $10 \mathrm{ml} \cdot \mathrm{kg}^{-1}$ d'une solution de Hartmann ont été perfusés ; ensuite, la vitesse de perfusion a été réduite à $2 \mathrm{ml} \cdot \mathrm{kg}^{-1} \cdot \mathrm{h}^{-1}$. L'espace péridural a été localisé au niveau $\mathrm{L}_{3} / \mathrm{L}_{4}$ selon la technique de la perte de résistance avec une aiguille de calibre $18(1,25 \mathrm{~mm})$ et $5 \mathrm{ml}$ d'une solution physiologique isotonique, en position latérale. Un cathéter de calibre $20(0,9 \mathrm{~mm})$ a été introduit en direction céphalique et fixé à 3 à $4 \mathrm{~cm}$ dans l'espace péridural. Pour un cas dans chaque groupe, l'espace $\mathrm{L}_{2} / \mathrm{L}_{3}$ a été ponctionné après un échec au niveau sous-jacent.

De la position latérale, les patients du premier groupe ont été remis en décubitus dorsal pour toute la durée de l'étude. Ceux du deuxième groupe ont été assis le buste vertical et les jambes allongées horizontalement, avant l'injection de la dose test. Ils ont conservé cette position pendant $15 \mathrm{~min}$ après l'injection de la dose totale avant d'être remis en décubitus dorsal pour le reste de l'étude. Tous les patients ont reçu une dose test de $3 \mathrm{ml}$ de lidocaïne $1,73 \%$ carbonatée fraichement adrénalinée à 1:200 000 et trois minutes plus tard, $17 \mathrm{ml}$ du même produit en deux minutes.

Un premier observateur a évalué l'installation de l'anesthésie péridurale toutes les trois minutes, pendant les 15 min suivant l'injection de l'anesthésique local. Un deuxième observateur, ignorant la position initiale, a poursuivi l'évaluation au même rythme pendant $30 \mathrm{~min}$. Seul le côté opéré a été testé afin d'assurer des mesures précises et rapprochées. L'extension céphalique et caudale du bloc sensitif a été évaluée par la disparition de la piqûre à l'aide d'une aiguille de calibre $24(0,55 \mathrm{~mm})$ (pin prick). Le niveau de discrimination thermique, représentatif $d u$ niveau du bloc sympathique, a été déterminé au moyen du test à l'éther. Les racines nerveuses $L_{1}$ à $S_{2}$ ont été testées aux dermatomes segmentaires usuels. Le bloc moteur a été évalué selon l'échelle proposée par Seow, ${ }^{16}$ modifiée pour un seul membre, de 0 à 5 d'après les myotomes. Une hypotension importante, définie comme une diminution de plus de $30 \%$ de la tension artérielle systolique initiale, et/ou une bradycardie de moins de $40 / \mathrm{min}$ ont été traitées par 5-10 mg d'éphédrine et/ou 0,5 mg d'atropine en injection intraveineuse, répétée si nécessaire.

Aucun sédatif ni analgésique n'a été administré pendant les premières $45 \mathrm{~min}$. Par la suite, si une sédation supplémentaire était jugée nécessaire, $1,5-3 \mathrm{mg}$ de midazolam étaient administrés par voie intraveineuse. Toutes les interventions ont été effectuées avec un garrot placé au niveau de la cuisse. Si le bloc sensitif était insuffisant dans la zone à opérer $45 \mathrm{~min}$ après l'injection d'anesthésique local, $6 \mathrm{ml}$ de la même solution étaient réinjectés. En cas de douleurs lors de l'incision, $100 \mu \mathrm{g}$ de fentanyl intraveineux furent administrés.

Les données paramétriques sont exprimées en moyenne \pm écart type et les données non paramétriques en médiane avec écart maximal. Les deux groupes ont été comparés, respectivement, avec le test de t et le test de Mann-Whitney ou du chi au carré. Un $P<0,05$ a été considéré comme significatif.

\section{Résultats}

Dans le groupe assis, un patient a été exclu de l'étude en raison d'une hypotension artérielle brutale et sévère, survenue trois minutes après l'injection de la dose totale, ce qui nous a obligé à le recoucher. Les paramètres hémodynamiques de ce patient se sont rapidement normalisés et l'anesthésie péridurale a été suffisante pour effectuer l'opération.

Les deux groupes étaient comparables pour l'âge (33 \pm 9) le poids $(68,5 \pm 12)$, la taille $(170 \pm 9)$, le côté opéré, le sexe et les paramètres hémodynamiques. De plus, il n'y a pas eu de différence significative entre les deux groupes pour le temps séparant le début de l'anesthésie de la chirurgie, la durée de l'intervention et la durée de gonflement du garrot. Le volume moyen administré de solution de Hartmann a été de $1375 \mathrm{ml}$ pour les deux groupes. 


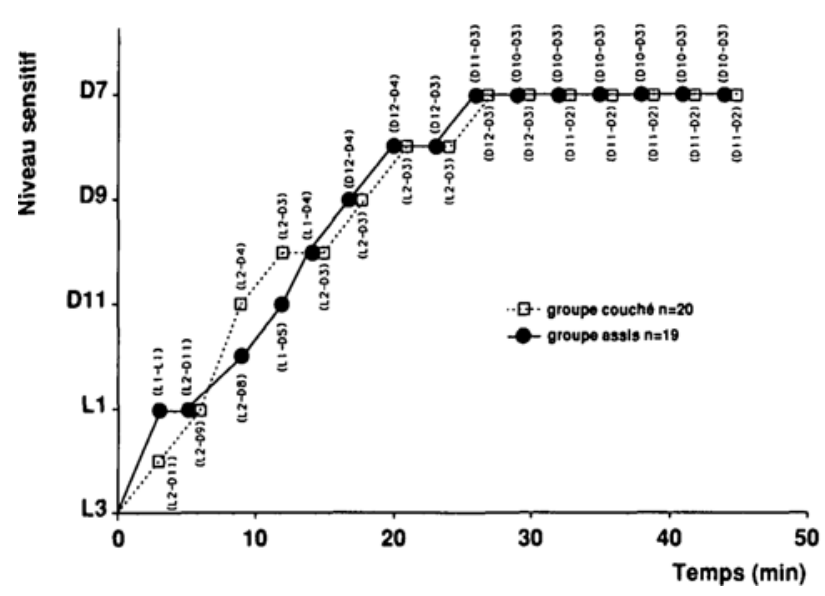

FIGURE 1 Progression céphalique du niveau sensitif pendant les 45 premières minutes (médiane; écart maximal entre parenthèse).

Bloc sensitif et sympathique

Le temps d'installation du bloc sensitif et sympathique pour les racines $L_{1}$ à $S_{2}$, est comparable pour les deux groupes. Il a débuté par les racines $L_{1}$ et $L_{2}$, avec un temps de latence plus important pour les racines $L_{5}$ et $S_{1}$ mais sans différence statistique entre les deux groupes. Cornme l'illustre la Figure 1, la progression céphalique du bloc sensitif a été semblable dans les deux groupes. Le niveau céphalique sensitif et sympathique maximum a été observé à $\mathrm{D} 7$ dans les deux groupes avec des écarts maximaux de D11 à D2 dans le groupe couché ; respectivement, D1 1 à D3 et D10 à D3 dans le groupe assis. Cependant, le niveau céphalique sympathique maximum a été atteint significativement plus rapidement dans le premier groupe en 22 $\pm 8 \mathrm{~min}$ contre $28 \pm 7 \mathrm{~min}$, ainsi que le niveau sensitif maximum $23 \pm 9 \min$ contre $29 \pm 6 \min (P<0.05)$.

\section{Bloc moteur}

L'installation du bloc moteur est illustrée dans la Figure 2. Jusqu'à 33 min après injection de l'anesthésique local, la valeur médiane du bloc moteur est comparable dans les deux groupes, puis, augmente significativement en position assise $(P<0,05)$. Après $42 \mathrm{~min}$, le bloc moteur n'a plus progressé dans les deux groupes.

La répartition des patients en fonction du degré du bloc moteur observé après 45 min est indiquée dans la Figure 3. Quatorze patients du groupe assis ont eu un bloc moteur $\geq 3$ contre seulement cinq en position couchée $(P<0,01)$.

\section{Variations hémodynamiques}

Les modifications hémodynamiques sont indiquées dans le Tableau. La diminution de la tension artérielle a été significativement plus importante et plus précoce en position assise. Alors que la fréquence cardiaque a aug-

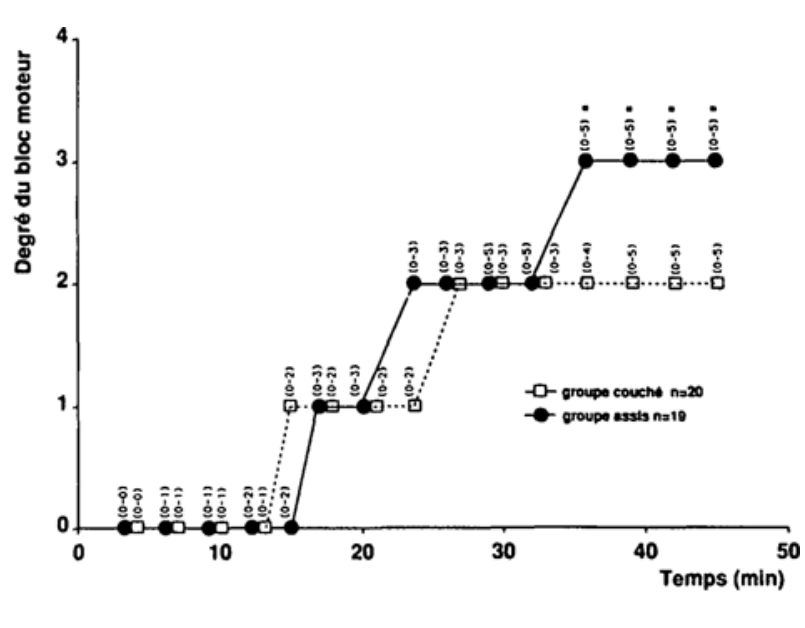

FIGURE 2 Evolution du degré de bloc moteur pendant les 45 premières minutes (médiane; écar maximal entre parenthèses), ${ }^{*} P<0,05$ par rapport au groupe couché.

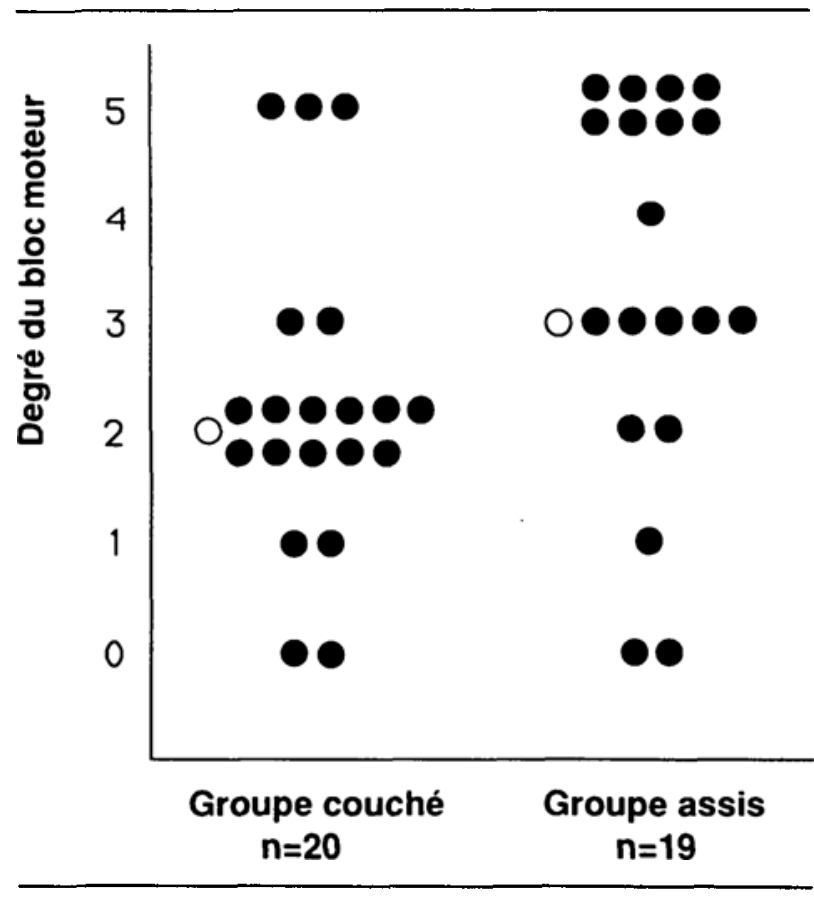

FIGURE 3 Répartition des patients selon le degré de bloc moteur dans les 2 groupes aprè les 45 premières minutes. - individuelle ; O médiane.

menté de $16 \%$ dans le groupe couché, elle a diminué de $5 \%$ dans le groupe assis $(P<0,05)$, la variation maximale survenant après un temps comparable. Il n'y a pas de différence significative pour le nombre de patients ayant reçu de l'atropine et/ou de l'éphédrine, ainsi que pour les doses moyennes administrées, ce qui est indiqué dans le Tableau. 
TABLEAU Changements hémodynamiques maximaux observés pendant les 45 premières minutes (moyenne \pm écart type)

\begin{tabular}{|c|c|c|}
\hline & $\begin{array}{l}\text { Groupe } \\
\text { couché } \\
n=20\end{array}$ & $\begin{array}{l}\text { Groupe } \\
\text { assis } \\
n=19\end{array}$ \\
\hline Diminution maximale de la TAM (\%) & $-16 \pm 10$ & $-24 \pm 10^{*}$ \\
\hline Observée après (min) & $22 \pm 10$ & $14 \pm 9 \dagger$ \\
\hline Variation maximale de la FC (\%) & $+16 \pm 26$ & $-5 \pm 30^{*}$ \\
\hline Observée après (min) & $19 \pm 12$ & $17 \pm 13$ \\
\hline $\begin{array}{l}\text { Nombre de patients ayant } \\
\text { reçu de l'éphédrine }\end{array}$ & $2 / 20$ & $5 / 19$ \\
\hline Dose moyenne d'éphédrine (mg) & $5 \pm 0$ & $9 \pm 2,2$ \\
\hline $\begin{array}{l}\text { Nombre de patients ayant } \\
\text { reçu de l'atropine }\end{array}$ & $2 / 20$ & $2 / 19$ \\
\hline Dose moyenne d'atropine $(\mathrm{mg})$ & $0,5 \pm 0$ & $0,5 \pm 0$ \\
\hline
\end{tabular}

$* P<0,05$.

$\dagger P<0,01$ par rapport au groupe couché.

$\mathrm{TAM}=$ tension artérielle moyenne. $\mathrm{FC}=$ fréquence cardiaque.

\section{Qualité de l'anesthésie}

Après l'injection initiale, l'anesthésie a été suffisante pour effectuer l'opération dans tous les cas. Toutefois, un patient du groupe assis a reçu $100 \mu \mathrm{g}$ de fentanyl intraveineux en raison d'un inconfort après l'incision. Tous les patients ont bien supporté le garrot, sauf pour un patient du groupe couché qui s'est plaint de sensation de brûlure et a été soulagé par l'injection intraveineuse de $100 \mu \mathrm{g}$ de fentanyl. Trois patients de chaque groupe ont reçu du midazolam à la dose totale moyenne de $2,8 \pm 1,3 \mathrm{mg}$ pour chaque groupe.

\section{Discussion}

Alors que pour plusieurs auteurs, la position assise lors d'une anesthésie péridurale influence favorablement les caractéristiques du bloc sensitif des racines lombosacrées, ${ }^{1,2,11,12}$ les résultats de notre étude n'ont pas permis de mettre en évidence un quelconque avantage, ce qui a déjà été rapporté par d'autres études. ${ }^{13,15}$

Quant à l'effet de la position assise sur le niveau céphalique maximum, des études antérieures ont rapporté des résultats contradictoires ; il était soit plus élevé, ${ }^{10}$ soit plus bas. ${ }^{11}$ Selon les études récentes, ${ }^{13-15}$ il apparaît que la position n'a pas d'effet sur ce niveau, comme c'est le cas pour notre étude. Seulement, le temps d'installation du niveau céphalique maximum a été significativement plus long en position assise, ce qui a déjà été décrit auparavant. ${ }^{15}$ Mais, ceci n'a pas eu de conséquences pour les interventions au niveau des membres inférieurs. Des différences méthodologiques importantes pourraient expliquer la divergence des résultats entre ces différentes études. En effet, la position assise a été conservée pendant un temps variant de 3 à $20 \mathrm{~min},{ }^{1,12-15}$ plusieurs anesthésiques locaux ont été utilisés et à des doses diffé- rentes. ${ }^{1,10,12-15} \mathrm{D}$ 'autre part, plusieurs études ont été réalisées en obstétrique, ${ }^{12-14}$ ce qui modifie la distribution des anesthésiques locaux dans l'espace péridural. ${ }^{1,2,17}$ Dans la présente étude, le bloc moteur a été amélioré en position assise. Ainsi, $70 \%$ des patients ont présenté un bloc moteur $\geq 3$ contre $25 \%$ seulement dans le groupe couché $(P<$ 0,01 ). Bien que moins important que le bloc sensitif à notre avis, un bloc moteur de meilleure qualité optimalise les conditions chirurgicales par l'absence de mouvements intempestifs du patient, lors de la réduction et l'ostéosynthèse d'une fracture par exemple.

L'amélioration de la qualité du bloc moteur des racines $L_{5}$ et $S_{1}$ en position assise suggère une meilleure imprégnation de ces racines par l'anesthésique local. Ceci a été postulé par Cousins et Bromage, ${ }^{2}$ bien que la gravité ne semble influencer que peu ou pas la distribution des anesthésiques locaux dans l'espace péridural. ${ }^{2,17} \mathrm{~L}$ 'utilisation de la lidocaïne carbonatée dans cette étude pourrait avoir masqué cet effet sur le bloc sensitif par sa capacité de diffusion élevée d'une part et la nécessité d'une concentration minimale d'anesthésique local moindre pour bloquer les fibres sensitives par rapport aux fibres motrices d'autre part. ${ }^{2}$ Le niveau sensitif céphalique maximum comparable entre les deux groupes pourrait être expliqué par une dose unique relativement élevée. En effet, il a aussi été suggéré que l'influence de la position assise serait plus marquée avec des doses plus faibles. ${ }^{4}$

Le bloc sensitif produit par la lidocaïne carbonatée a été de bonne qualité chez tous les patients, pour les racines $L_{5}$ et $S_{1}$ en particulier, ce que plusieurs auteurs ont déjà montré. ${ }^{4-6}$ Seulement deux patients ont présenté un malaise à l'incision ou au niveau du garrot, traité efficacement par $100 \mu \mathrm{g}$ de fentanyl intraveineux.

La diminution de la tension artérielle moyenne a été significativement plus importante et plus précoce dans le groupe assis vraisemblablement en raison de l'orthostatisme. La différence significative entre la baisse de $5 \%$ de la fréquence cardiaque en position assise et l'augmentation de $16 \%$ en décubitus dorsal peut être expliquée par une augmentation du tonus vagal dans le deuxième groupe, masquant l'effet systémique de l'adrénaline ajoutée aux anesthésiques locaux. ${ }^{2}$ Elle aurait pour origine une diminution du retour veineux due à l'anesthésie péridurale ${ }^{18}$ et la position assise.

Dans les deux groupes, les temps d'installation ainsi que les niveaux céphaliques sensitif et sympathique maximaux sont similaires. Ces résultats confirment l'absence de différence entre le niveau sensitif et sympathique pour l'anesthésie péridurale. ${ }^{19}$

En conclusion, cette étude confirme l'efficacité de la lidocaïne carbonatée adrénalinée pour les racines $L_{5}$ et $S_{1}$ lors d'une anesthésie péridurale. Avec la dose utilisée, la position assise n'a pas eu d'influence sur le bloc sensitif 
mais a amélioré significativement la qualité du bloc moteur et a provoqué une diminution significativement plus importante de la tension artérielle moyenne toutefois sans atteindre de valeurs critiques.

\section{References}

1 Bromage PR. Epidural Analgesia. Philadelphia: WB Saunders, 1978; 484-512, 513-600.

2 Cousins MJ, Bromage PR. Epidural neural blockade. In: Cousins MJ, Bridenbaugh PO, (Eds.). Neural Blockade in Anesthesia and Management of Pain, 2nd ed. Philadelphia: JB Lippincott, 1988; 253-360.

3 Galindo A, Hernandez J, Benavides $O$, Ortegon de Munoz $S$, Bonica JJ. Quality of spinal extradural anaesthesia: the influence of spinal nerve root diameter. $\mathrm{Br} \mathrm{J}$ Anaesth 1975; 47: 41-7.

4 Bromage $P R$. Physiology and pharmacology of epidural analgesia. Anesthesiology 1967; 28: 592-622.

5 Park WY, Hagins FM. Comparison of lidocaine hydrochloride and lidocaine carbonated for epidural anesthesia. Regional Anesthesia 1986; 11: 128-31.

6 Nickel MP, Bromage PR, Sherrill DL. Comparison of hydrochloride and carbonated salts of lidocaine for epidural analgesia. Regional Anaesthesia 1986; 11: 62-7.

7 Martin R, Lamarche Y, Tetreault $L$. Comparison of the clinical effectiveness of lidocaine hydrocarbonated and lidocaine hydrochloride with or without epinephrine in epidural anesthesia. Can Anaesth Soc J 1981; 28: 217-23.

8 Morison DH. A double-blind comparison of carbonated lidocaine and lidocaine hydrochloride in epidural anaesthesia. Can Anaesth Soc J 1981; 28: 387-9.

9 Cole CP, McMorland GH, Alexson JE. Epidural blockade for caesarean section: lidocaine- $\mathrm{HCl}$ versus lidocaine$\mathrm{CO}_{2}$. Anesthesiology 1985; 62: 348-50.

10 Nishimura N, Kitahara T, Kusakafe T. The spread of lidocaine and $\mathrm{I}^{131}$ solution in the epidural space. Anesthesiology $1959 ; 20: 785-8$.

11 Bromage $P R$. Spread of analgesic solutions in the epidural space and their site of action: statistical study. $\mathrm{Br}$ J Anaesth 1962; 34: 161-78.

12 Thorburn J, Moir JJ. Epidural analgesia for elective caesarean section. Anaesthesia 1980; 35: 3-6.

13 Merry AF, Cross JA, Mayadeo SV, Wild CJ. Posture and the spread of extradural analgesia in labour. $\mathrm{Br} \mathrm{J}$ Anaesth 1983; 55: 303-6.

14 Park WY, Hagins FM, Massengale MD, MacNamara TE. The sitting position and anesthesic spread in the epidural space. Anesth Analg 1984; 63: 863-4.

15 Norris $M C$, Dewan $D$. Effect of gravity on the spread of extradural anaesthesia for caesarean section. Br J Anaesth 1987; 59: 338-41.
16 Seow LT, Lips FJ, Cousins MJ. Lidocaine and bupivacaine mixtures for epidural blockade. Anesthesiology 1982; 56: 177-83.

17 Park WY. Factors influencing distribution of local anesthetics in the epidural space. Regional Anaest 1988; 13 : 49-57.

18 Baron JF, Decaux-Jacolot A, Edouard A, Berdeaux A, Kamran $S$. Influence of venous return on baroreflex control of heart rate during lumbar epidural anesthesia in humans. Anesthesiology 1986; 64: 188-93.

19 Wugmeister M, Hehre FW. The absence of differential blockade in peridural anaesthesia. Br J Anaesth 1967; 39: 953-6. 